\title{
Germanica
}

\section{Persistance de la mémoire : le mal d'être dans la poésie de Rose Ausländer}

Beständigkeit der Erinnerung. Weltschmerz in der Lyrik Rose Ausländers

\section{Jacques Lajarrige}

\section{OpenEdition}

Journals

Édition électronique

URL : http://journals.openedition.org/germanica/2622

DOI : $10.4000 /$ germanica.2622

ISSN : 2107-0784

Éditeur

Université de Lille

\section{Édition imprimée}

Date de publication : 31 décembre 1989

Pagination : $29-40$

ISSN : 0984-2632

\section{Référence électronique}

Jacques Lajarrige, «Persistance de la mémoire : le mal d'être dans la poésie de Rose Ausländer », Germanica [En ligne], 5 | 1989, mis en ligne le 09 février 2015, consulté le 06 octobre 2020. URL : http:// journals.openedition.org/germanica/2622; DOI : https://doi.org/10.4000/germanica.2622

Ce document a été généré automatiquement le 6 octobre 2020.

(C) Tous droits réservés 


\title{
Persistance de la mémoire : le mal d'être dans la poésie de Rose Ausländer
}

\author{
Beständigkeit der Erinnerung. Weltschmerz in der Lyrik Rose Ausländers
}

Jacques Lajarrige

Es tut weh

und wohl

nicht zu vergessen ${ }^{1}$

1 Rose Ausländer, de son nom de jeune fille Rosalie Scherzer, est née le 11 mai 1901 à Tchernovtsy, l'ancienne capitale de la Bucovine (Czernowitz), alors province de l'empire austro-hongrois, dans une famille juive de langue allemande. Très tôt, son existence est marquée par la douleur. Douleur de l'exil tout d'abord, puisqu'en 1921, un an après la mort de son père, elle doit émigrer en Amérique, afin de soulager la situation matérielle précaire de sa famille. Rose Ausländer survivra tant bien que mal comme employée de banque tout en se consacrant à la poésie et au journalisme. Ses débuts lyriques sont marqués par un attachement formel et thématique au romantisme et à une diction toute rilkéenne. Mais d'autre part, la misère de la mégalopolis américaine lui inspire le cycle New York, qui la place dans la lignée des poètes expressionnistes ${ }^{2}$.

2 En 1931, Rose Ausländer regagne sa ville natale pour soigner sa mère gravement malade. Sur les conseils et avec l'aide d'amis américains qui la préviennent du danger nazi, elle quitte à nouveau la vieille Europe pour New York en mars 1939. Mais l'attachement à la mère reste plus fort que tout, et une rechute de cette dernière la décide à rentrer aussitôt auprès d'elle. C'est alors que le drame s'abat sur elle et les siens. À partir de 1941 et jusqu'en 1944, les nazis occupent la Bucovine et séquestrent les juifs dans un ghetto. Commencent alors l'isolement, les privations de nourriture, l'humiliation de l'étoile jaune et des travaux forcés. Pour échapper à la déportation, Rose Ausländer et sa mère devront se cacher dans des caves à partir de 1943. 
Ces douloureuses épreuves successives forment l'arrière-plan biographique de l'œuvre lyrique de Rose Ausländer. Dès lors, plus rien ne pourra être comme avant. Après la guerre commence pour la poétesse une deuxième et longue période d'exil en Amérique. Les persécutions nazies, la mort collective de millions de juifs, les images à jamais enracinées du ghetto auront inévitablement des répercussions tant thématiques que formelles sur l'œuvre. Le mal d'être de la femme survivante s'exprime en premier lieu par une interrogation: la poésie est-elle encore possible ou, plus justement, quelle poésie est licite. Face à l'horreur, le poète ne doit pas se taire, mais porter témoignage, telle sera la réponse de Rose Ausländer.

\section{Le paradis perdu de l'enfance}

4 De par sa naissance même, Rose Ausländer, juive de langue allemande qui ne grandit pas en Allemagne, se trouve $a b$ origine étrangère dans son pays natal. Ce déracinement est bien sûr directement lié à la situation géographique et historique de la Bucovine, ancienne province d'un empire dont l'existence cesse en 1918. De ce fait, Rose Ausländer perdra une « patrie » que sa naissance ne lui avait pas octroyée. Czernowitz désormais Cernauti - et la Bucovine deviennent roumaines, puis soviétiques après la Deuxième Guerre.

5 L'attachement farouche que les poèmes ne cesseront de marquer aux paysages idylliques de l'enfance délimités par le mont Raréu et le Pruth, fleuve qui prend chez elle une valeur quasi mythique, témoigne du sentiment douloureux d'avoir été dépossédée d'un bien irremplaçable. Par contraste avec les vues enchantées d'un monde lié à l'enfance, le double exil américain suscite un profond malaise. L'Américain Way of Live dévoile à la jeune femme la cruauté d'un monde industrialisé et mécanisé à outrance et ses laissés-pour-compte qui n'ont d'autre refuge que la rue et l'alcool. Perdue dans ce monde où, comme le dira plus tard le poème Das Dividierte Gedicht du recueil Blinder Sommer (1965), le «sourire électrique" tient lieu de bonheur, Rose Ausländer traduira avec force son sentiment d'être étrangère.

Pourtant, le retour au pays natal, provoqué à deux reprises, nous l'avons dit, par l'état de santé de la mère, ne signifiera jamais la reconquête du paradis perdu de l'enfance. Le retour prend avec l'insupportable calvaire du ghetto le sens d'un exil intérieur cette fois, dont les Gettomotive retraceront les différentes stations. Ces poèmes qui ne sauraient être jugés en fonction de leur qualité littéraire, marquent une rupture brutale et décisive dans la production du poète. Chassée de "Chez elle» tout en y étant revenue, la poétesse verra s'effondrer sous ses yeux l'image paradisiaque du pays conservée dans le souvenir. À partir de ce moment deux attitudes face à la Bucovine natale se dessineront, qui correspondent également à une évolution dans le temps. Le deuxième recueil de poèmes, Blinder Sommer, publié en 1965, marquera la tentative, notamment au travers du cycle Das Dorf Duminika, de soustraire définitivement les paysages de l'enfance à l'emprise du temps et de l'Histoire ${ }^{3}$. Il faut ici souligner que le retour de la poésie aux origines de la naissance ne peut être qu'un retour mental, soustrait aux contingences de tous ordres. Indéniablement, les paysages évoqués font corps avec les parents du poète. Le père apparaît comme le gardien de la tradition et de la mystique juives, notamment celle des Chassidim de Sadagora au milieu desquels il avait grandi et dont il avait reçu l'enseignement : 
Am Hof des Wunderrabbi von Sadagora

lernte der Vater die schwierigen Geheimnisse

Seine Ohrlocken läuteten Legenden

in den Händen bielt er den hebräischen Wald ${ }^{4}$.

Mais c'est, sans conteste la mère qui demeure l'une des figures ordonatrices les plus importantes de la poésie de Rose Ausländer. Celle que le poète désigne comme "son rossignol » (cf. le poème Meine Nachtigall, G.W., II, p. 317) détient à elle seule la clef de l'univers affectif et spirituel de l'œuvre. Comme le père, la mère relie l'enfant à l'histoire des ancêtres et donc du peuple juif depuis les origines. De plus, comme elle chante la vieille Autriche, elle valorise le lien de la Bucovine avec l'Empire déchu et offre, utopiquement du moins, la possibilité d'un retour qui fait fi des avatars de l'Histoire. En même temps, sa mort met un terme brutal à cette filiation et rejette irréversiblement dans l'espace froid et déshumanisé de l'exil. Essentiel contrepoids à l'isolement culturel de Rose Ausländer, repère fidèle parmi les effondrements successifs de son destin personnel, indissociable de celui du peuple juif, la mère assure dans l'ombre le complexe labeur d'un aiguillon de vie, d'une barrière contre une dépossession du monde et de l'identité. D'autre part, elle demeure celle qui, avec le dernier soupir rendu, fait basculer l'éternelle enfant, encore tout émerveillée par les couleurs et les parfums du pays natal, dans une angoissante solitude, la livrant au désespoir sans fond :

Gestern tauchte die

tote Mutter ein heller

Delphin aus dem Wasser

nahm mich nicht wahr ${ }^{5}$.

Partenaire privilégié, déchirure d'un décor que seul le rêve, aux prises avec les cauchemars, peut encore faire surgir à l'horizon, la mère reste indissociable du paysage bucovinien que les poèmes sentent plus qu'ils ne les représentent. Le paysage se plie ici aux attitudes des personnages qui le hantent. Mais surtout, avec l'évocation de la Bucovine et de la ville natale, Rose Ausländer ne se lasse pas d'arpenter par le souvenir le monde perdu de l'enfance qu'elle cherche sans cesse à retrouver comme s'il devenait ainsi le sein maternel immortalisé, havre de paix où l'égarée dans la tourmente peut se recoquiller et retrouver, l'espace et le temps réduits d'un poème, une chaude et sécurisante intimité.

Pourtant, la mort de la mère condamne le poète à l'errance, qui trouvera son expression poétique dans de nombreuses formulations antithétiques : «in der Fremde daheim» (Daheim, G.W., VI, p. 27) ; «Heimgekehrt in die Fremde» (Ibid., p. 23); «heimatfremde» (G.W., IV, p. 128). En effet, le Pruth et le Raréu ne sont pas seulement les dépositaires du souvenir idéalisé de l'enfance, ils sont devenus contre leur gré les témoins de l'horreur perpétrée contre les juifs de Bucovine par les nazis d'abord, puis par les «Libérateurs" soviétiques qui, après avoir libéré le ghetto de Czernowitz, déporteront les rescapés dans des camps de travail sous prétexte qu'ils sont «allemands ». Le retour défendu dans ce qui finira par devenir dans Mein Atem heisst jetzt la «terre interdite» (G.W., VI, p. 181) contraindra le poète à la recherche d'un autre refuge, celui de la langue. 


\section{Langue perdue, langue retrouvée}

10 La nostalgie des origines, l'impossibilité de les retrouver, le langage poétique de Rose Ausländer en épouse tous les méandres, en répercute la douleur. Il se trouve que l'harmonie de la Bucovine résidait également dans le mélange des cultures et des langues comme se plaisent à le souligner plusieurs poèmes. Les hymnes aux paysages de l'enfance sont eux-mêmes à considérer comme des vestiges sonores d'anciennes harmonies brisées, comme l'écho d'un monde où la parole, dans la plurivocité de ses manifestations linguistiques, témoignait de la souveraineté du cœur et de la raison (cf. le poème Czernowitz, G.W., VI, p. 346). Le Verbe était encore au service de la construction pacifique du monde et non de sa destruction. Ce-disant, Rose Ausländer ne traite pas à proprement parler du thème de la langue, elle l'évoque comme une empreinte effacée par l'Histoire et la folie destructrice des nazis.

11 Pourtant, une réflexion sur le langage s'amorce dans l'œuvre, qui permettra de mieux mesurer le tragique destin de celle pour qui le retour ne peut s'accomplir par une direction géographique, mais seulement par le douloureux cheminement du souvenir. La langue, par le biais du travail poétique, jette une lumière cruelle sur sa vie. Elle lui fait prendre conscience que son pays natal n'a plus d'existence que mentale et, partant, immatérielle. D'autre part, en en faisant l'un des thèmes récurrents de son univers lyrique, elle lui redonne du même coup un espace qui est celui du matériau poétique imprimé :
Mein Vaterland ist tot
sie haben es begraben
im Feuer
Ich lebe
in meinem Mutterland Wort ${ }^{6}$.

Le lien vernaculaire qui unit la langue à la mère, exprimé ici avec force par le "Mutterland Wort », indique clairement que toutes deux participent à la fois du lieu originaire perdu et du nouveau lieu à conquérir que sera le poème.

Ressaisi dans cette perspective, le thème du retour pose, obliquement, celui du retour à la langue et se trouve à la source de la reconquête vitale d'un site habitabe ${ }^{7}$. Le langage devient alors l'objet d'un discours paradoxal qui affirme la difficulté de dire, mais qui jette aussi les bases d'une nouvelle voie (voix !) poétique qui, timidement d'abord, dans un concert souvent silencieux, criblé de cris de détresse et de chuchotements d'espoir, cherche à regagner du terrain sur le malheur et la souffrance. N'oublions pas que de 1946 à 1956, Rose Ausländer connaîtra le prix de ce silence et ne pourra plus écrire des poèmes qu'en anglais. Bien sûr, la proximité avec la douleur physique et intellectuelle fait résonance à ce doute. Le deuxième poème des Gettomotive, intitulé Fäden ins Nichts gespannt (G.W., I, p. 152) affirme que la menace de la mort rend vaine toute tentative d'embellissement du monde par l'expression métaphorique. Le poète est ce tisserand qui « tend des fils dans le néant » et, ce faisant, renforce cruellement son désespoir, on connaît l'affirmation d'Adorno pour qui il est impossible d'écrire des poèmes après Auschwitz, puis l'atténuation de ce jugement sévère qui rend licite toute poésie qui tient compte d'Auschwitz. Avec le recul, la position de Rose Ausländer évoluera elle aussi dans ce sens. À partir de Blinder Sommer se fait jour une nouvelle conception de la 
langue qui s'oppose au célèbre «Kristall in der Tracht deines Schweigens » de Paul Celan :

Komm die Dünen sind heute

tönend und transparent :

eine küste aus ruf und Kristall ${ }^{8}$.

La confiance retrouvée dans la force du poème ne signifie pas pour autant à ses yeux que le poète peut se contenter d'écrire comme si rien ne s'était passé. Le langage de Rose Ausländer s'instituera dans cet intervalle ambigu et étroit entre la puissance du Verbe divin et le souffle ténu des survivants, dans ce compromis entre la difficulté de l'impérieux témoignage et l'exigence éthique de la "transparence ». Redécouvrir la faculté de parole, c'est pour Rose Ausländer pénétrer dans un no man's land qui s'étend à perte de vue entre soi-même, le monde perdu de l'enfance et autrui. Mais, similairement, c'est s'aventurer dans un univers neuf où l'acte de parole se fera reconquête d'un sens dont les événements historiques avaient gommé toute trace. D'un point de vue esthétique, cette reconquête s'accompagne d'un bouleversement profond de la diction poétique, d'une remise en question des formes traditionnelles et de l'abandon de la rime au profit des rythmes libres'. Enfin, et surtout, oubliant la langue des bourreaux galvaudée par la propagande raciale, il s'agira pour le poète d'inventer un «Nouveau calendrier, un nouvel alphabet, une langue qui mette un terme " (cf. Nicht Oktober nicht November, G.W., II, p. 298). Un terme à l'horreur, à la haine et au racisme.

On comprend mieux dès lors à quelle immense tâche s'attèle l'œuvre de Rose Ausländer, et on comprend mieux aussi qu'une telle tâche puisse susciter une forme particulière de désespoir. La langue retrouvée devient en effet le point de cristallisation d'une expérience du monde marquée par la bipolarité : l'exil et l'impossible retour au pays natal, le silence absolu ou le mot compris comme «Wiederwort», c'est-à-dire à la fois comme renaissance à la vie et douloureux rappel du passé, mais dans tous les cas comme un «Atemwor $\mathrm{t}^{10}$ », une bouffée d'oxygène qui évite que le passé ne vous étouffe complètement. En ce sens, l'écriture témoigne d'une recherche angoissée de l'identité perdue et à venir. Le mot, ce phénix surgi des urnes funéraires où repose la mémoire des juifs exterminés dans les camps, sauve de l'oubli en même temps qu'il appelle à une vie nouvelle.

\section{Le souvenir des morts}

Même s'il est vrai, comme le dit laconiquement Paul Celan, que « nul ne témoigne pour le témoin ${ }^{11}$ ", la situation historique de Rose Ausländer, tout à la fois victime juive et rescapée, ne pouvait manquer de marquer sa démarche d'écrivain. Avoir échappé à la mort collective fait naître un sentiment de culpabilité :

Tote Freunde

klagen dich an

du hast sie überlebt ${ }^{12}$

et paradoxalement le désir de mourir soi-même, comme pour expier une faute qui ne peut en être une :

Ich werde hundert Jahre sterben

ich bin bereit ${ }^{13}$. 

répétition par laquelle il espère pouvoir se charger du lourd fardeau de la mort des siens. Pourtant, plus secrètement, l'expérience poétique s'enracine dans cet impossible dépassement physique, dans l'assimilation de la mort d'autrui. Par un phénomène rédempteur d'osmose avec les défunts, la poésie se placera désormais sous le signe de la remémoration et investira le poète d'une mission éthique. Alors, mais alors seulement, dans un mouvement dialectique inversé cette fois, la mort pourra devenir le lieu du retour à la vie comme le suggère le poème Urne Erinnerung:

Nichts verloren

In der Urne

die Asche atmet ${ }^{14}$

Sur un même plan, la résurrection des morts, métaphoriquement rendus à la vie dans le temps du discours, permet d'oublier un moment qu'on n'est qu'un mort-vivant. Mais cette résurrection demeure éphémère, Rose Ausländer le sent bien du reste, et doit pour cette raison être sans cesse renouvelée. Ainsi, l'écriture ne peut plus seulement se vouloir mémoire, elle se doit, pour répondre aux exigences existentielles qui en impose la dictée, de parcourir toujours et encore le chemin de croix des victimes. L'image des souvenirs passés au laminoir («Erinnerungswalze», in G. W., IV, p. 219) traduit bien cette lancinante douleur de la souvenance. Ici, la mémoire est l'absence que la présence du poème supplée. Parallèlement se dessine l'idée que le futur et le passé s'abolissent et que c'est le futur qui « dicte des poèmes à la mémoire » (cf. le poème Gedächtnis II, G.W., IV, p. 20). Dans le dialogue qui s'instaure entre le poète et les victimes, s'inscrit en filigrane la conviction que le souvenir ne peut se résumer à une attitude passéiste, à un regard mélancolique sur le pays des origines. Au regard de l'Histoire, la persistance de la mémoire transcende les oppositions temporelles et place le poème sur la voie de la réconciliation des hommes. A partir de là, la vocation dialogique de l'œuvre prendra tout son sens.

20 Cependant, les morts se taisent comme le répètent de nombreux poèmes. Meine Toten schweigen tief dit en substance l'un des cycles du recueil Ich spiele noch. Et quand bien même des signes nouveaux se manifesteraient, personne ne saurait les interpréter. Leur silence voue les survivants à la solitude du deuil.

\section{La mort du souvenir?}

21 D'avril à décembre 1957, Rose Ausländer entreprend un long voyage à travers l'Europe qui la conduit en France, en Espagne, en Italie, puis en Suède où elle rend visite à Nelly Sachs. Cette nouvelle manière de reconquérir le monde compense à n'en pas douter l'impossibilité de retour en Bucovine. L'œuvre porte à partir de ce moment témoignage d'une nouvelle perception de l'Histoire, positive par rapport au passé récent, notamment au travers des poèmes de Blinder Sommer consacrés à l'Italie (Offener Brief an Italien, Venedig, Villa d'Esté...) dans lesquels on reconnaît la marque constructive de l'homme de culture en tout opposé aux barbares nazis.

En décembre 1961, Rose Ausländer doit abandonner définitivement son travail à New York. Peu à peu, l'idée d'un retour en Europe germe en elle. En 1963, elle décide de rentrer en Autriche, puis traverse à nouveau l'Italie et l'Espagne. Mais c'est en fin de compte à Düsseldorf qu'elle s'installera définitivement en 1965. Commence alors un long processus de réclusion forcée que la maladie rendra irréversible. L'évolution de sa 
production ira elle aussi dans le sens d'un retrait progressif du monde, marqué essentiellement, nous l'avons vu, par la mort et la douleur du passé à jamais perdu. La vie n'est plus possible que dans les légendes et les rêves qui prendront dans les derniers recueils importants Mein Atem heisst jetzt, Mein Venedig versinkt nicht, Ich spiele noch, le pas sur la réalité.

La maladie qui condamne le poète à l'enfermement dans une chambre du foyer « Nelly Sachs ", la maison de retraite juive de Düsseldorf, la privera à partir de 1978 de tout contact direct avec le monde extérieur. Seules les étoiles la guident dans la nuit, seul le chant des oiseaux parvient jusqu'à elle comme une voix d'outre-monde. Parallèlement à la réduction de l'espace physique, la langue s'affranchit au fur et à mesure des référents concrets pour tendre à une forme d'abstraction et d'apesanteur qui traduit une ultime tentative de recouvrer la liberté. Le refuge dans le pays natal lui est interdit, le refuge dans la langue, à mesure que le poète la reconquiert, ravive la douleur de l'absence. Puis, dans un dernier soubresaut, le langage devient réflexion sur lui-même (cf. Das Wort, G.W. VI, p. 140). Mais, dans la mesure où la langue tout d'abord reconquise pour la conjuration de l'holocauste se perd dans la circularité du dire qui fait d'elle un métalangage, elle menace d'étouffer le souffle ténu qui la maintenait en vie.

La fuite dans les étoiles, échappatoire mentale du poète grabataire, construit un dernier pont entre le passé et le futur. L'étoile jaune, signe d'asservissement, devient nouvelle liberté et scelle la tendance à l'universalité du destin du peuple juif au service de laquelle la mémoire avait placé ses forces intimes. Ainsi se concrétise le vœu secret d'une régénérescence cosmique à la mesure de l'anéantissement de masse.

Paradoxalement, cette dernière envolée dans les étendues interstellaires accomplit le même trajet que la fumée des camps de la mort, dans une aspiration ascendante qui réunit les morts-vivants et les morts dans un ailleurs dont l'immatérialité céleste abolit toute notion d'espace et de temps et annihile toute idée de haine ou de vengeance. Mais l'infinitude du firmament, contrecarrant les limites tracées par l'enfermement clinique, menace en dernière analyse l'efficacité du combat mené contre l'oubli collectif. Quelle trace subsistera de cette dissolution parfaite? Qui désormais entendra le miserere du poète, sussurré dans le doute?

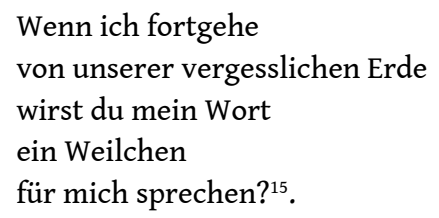

Atteinte d'une grave maladie, Rose Ausländer s'est éteinte au début de l'année 1988 à l'âge de 87 ans au foyer "Nelly Sachs". Son œuvre, immense et émouvante par sa vérité et sa simplicité, nous reste comme un authentique message d'humanité et de paix, comme une exhortation à témoigner à notre tour. 


\section{NOTES}

1. - Sauf mention contraire, les poèmes cités renvoient à l'édition complète des poèmes de Rose d'Ausländer, établie par Helmut Braun, Gesammelte Werke in sieben Bänden, S. Fischer Verlag, Frankfurt am Main. Nous indiquons chaque fois l'abréviation G.W., suivie de la référence du volume et de la page.

G. W., VI, P. 88 :

Cela fait du mal

et du bien

de ne pas oublier.

2. - Le titre d'un des poèmes du cycle New York (1927), Der Dämon der Stadt, fait explicitement référence à Georg Heym.

3. - Voir à ce propos les poèmes « Das Dorf Duminika, Kindheit I, Sukkoth, Pruth, Raréu, Dorf in der Bukowina », in G. W., II, respectivement p. 316, 321, 323, 324, 325 et 327.

4. - Cf. «Der Vater», in G.W., II, p. 318 :

À la cour du rabbi de Sadagora

le père apprit les difficiles secrets

Ses papillotes résonnaient de légendes

dans ses mains il tenait la forêt hébraïque.

5. - Cf. Stürmische Nacht, ibid., p. 295 :

Hier ma défunte mère

un dauphin clair

sortit de l'eau

ne me remarqua pas.

6. - Cf. « Mutterland », in G. W., p. 98 :

Ma patrie est morte

ils l'ont enterrée

dans le feu

Je vis

dans ma matrie

le Verbe

7. - C'est là une expérience commune à d'autres poètes juifs, notamment à Paul Celan, natif de Czernowitz comme Rose Ausländer, et à Nelly Sachs. Concernant l'œuvre de cette dernière et la thématique du refuge, nous renvoyons à l'article de G. Ueberschlag, « Nelly Sachs ou le refuge de la langue », Germanica 1/1987, p. 91-106.

8. - Cf. « Ruf und Kristall », in G.W., t. II, p. 262 :

Viens les dunes sont aujourd'hui

sonores et transparentes

une côte de cris et de cristal.

9. - Nous savons, grâce au court récit autobiographique «Alles kann Motiv sein » (G. W., III, p. 287), que les deux rencontres avec Paul Celan à Paris en juin et novembre 1957 constituèrent pour Rose Ausländer un choc esthétique durable. La lecture de Sprachgitter eut des répercussions sur son écriture qui ignorait alors tout de l'évolution de la poésie moderne de langue allemande.

10. - On trouve des variations sur ce même thème du Verbe compris comme souffle dans les poèmes «Ich suche » (G. W., V, p. 110). « Sang und Ozon » (G. W., II, p. 266) et « Raum II », (G. W., IV, p. 221).

11. - Paul Celan, «Aschenglorie», $m$ Atemwende, Suhrkamp Verlag, Frankfurt am Main 1967, p. 68 : « Niemand / zeugt für den / Zeugen». 
12. - G.W., IV, p. 182 :

Des amis défunts

t'accusent

tu leur as survécu.

13. - Cf. « Trage mich » in Ich spiele noch, S. Fischer, Frankfurt am Main, 1987, p. 108 :

je mourrai

pendant cent ans

je suis prête.

14. - G.W., III, p. 54 :

Rien perdu

dans l'urne

la cendre respire.

15. - G.W., VI, p. 19 :

Lorsque je quitterai

notre monde oublieux

parleras-tu toi mon Verbe

un instant

en ma faveur?

\section{RÉSUMÉS}

Juive de langue allemande, née dans l'ancienne capitale de la Bucovine, Czernowitz Rose Ausländer connaîtra par deux fois l'exil en Amérique, puis de 1941 à 1944 dans sa ville natale, l'exil intérieur du ghetto où elle dut porter l'humiliante étoile jaune et se terrer dans des caves avec sa mère malade pour échapper à la déportation.

Dès lors, son œuvre sera placée sous le signe du souvenir: souvenir idéalisé du pays natal inaccessible autrement que par le rêve et par l'évocation de la mère, souvenir des morts dont la souffrance dicte aux survivants le devoir éthique de témoigner. Mais le témoignage ne va pas de soi, il s'accompagne d'une grave crise de confiance dans la force du Verbe. Prise entre la difficulté de dire l'horreur qui ravive le mal d'être et la nécessité de sauver son propre passé et les victimes de l'oubli, le poète inventera un «nouvel alphabet» régi par la volonté de réconcilier les hommes.

Avec l'âge et la maladie, le langage poétique de Rose Ausländer deviendra de plus en plus abstrait jusqu'à en devenir métalangage, oubliant ainsi les prémisses de sa renaissance. Le " mot souffle » menace à nouveau de s'éteindre.

Rose Ausländer, 1901 in Czernowitz -, der ehemaligen Hauptstadt der Bukovina - als Kind deutschsprachiger Juden geboren, musste zweimal in die U.S.A. auswandern und erlebte zwischen 1941 und 1944 in ihrer Heimtstadt das innere Exil des Ghettos, wo sie den erniedrigenden Judenstern tragen musste und sich mit der kranken Mutter in Kellern versteckte, um der Deportation zu entkommen.

Ihre Lyrik steht fortan im Zeichen der Erinnerung; Erinnerung an den idealisierten Gedankenort Heimat, zu dem nur noch Traumvisionen und die Heraufbeschwörung des Mutterbildes Zugang verschaffen, aber auch Erinnerung an die jüdischen Opfer des Nationalsozialismus, deren Leid den Überlebenden die ethische Pflicht auferlegt, Zeugnis abzulegen. 
Doch schon bald nach dem Zusammenbruch stellen sich Zweifel an der Kraft des Wortes ein. Hin und her gerissen zwischen der Schwierigkeit, die Greueltaten sichtbar zu machen, und der lebenswichtigen Notwendigkeit, die eigene Vergangenheit sowie die Toten der Vergessenheit zu entreissen, wird Rose Ausländer schliesslich «ein neues Alphabet» erfinden, das den Willen bekundet, die Menschen miteinander zu versöhnen.

Kennzeichnend für den Altersstil Rose Ausländers ist, dass nun die abstrakten Begriffe die Worte der Rückbesinnung auf das verlorene Paradies der Kindheit allmählich verdrängen. Das «Atemwort» droht wieder zu ersticken. Astrid STARCK, Eine Zeit in Galizien: Autopsie einer Sprache. Über Die Galizianerin von Brigitte Schwaiger und Eva Deutsch.

\section{AUTEUR}

\section{JACQUES LAJARRIGE}

Université de Clermont II 